\section{Can Light Emitted from Smartphone Screens and Taking Selfies Cause Premature Aging and Wrinkles?}

\author{
Arjmandi N. ${ }^{1}$, Mortazavi Gh. ${ }^{1}$, Zarei S. ${ }^{2}$, Faraz M. ${ }^{3}$, Mortaza- \\ vi S. A. R. ${ }^{*}$
}

\begin{abstract}
Since the early days of human life on the Earth, our skin has been exposed to different levels of light. Recently, due to inevitable consequences of modern life, humans are not exposed to adequate levels of natural light during the day but they are overexposed to relatively high levels of artificial light at night. Skin is a major target of oxidative stress and the link between aging and oxidative stress is well documented. Especially, extrinsic skin aging can be caused by oxidative stress. The widespread use of light emitting diodes (LEDs) and the rapidly increasing use of smartphones, tablets, laptops and desktop computers have led to a significant rise in the exposure of human eyes to short-wavelength visible light. Recent studies show that exposure of human skin cells to light emitted from electronic devices, even for exposures as short as 1 hour, may cause reactive oxygen species (ROS) generation, apoptosis, and necrosis. The biological effects of exposure to short-wavelength visible light in blue region in humans and other living organisms were among our research priorities at the Ionizing and Nonionizing Radiation Protection Research Center (INIRPRC). Today, there is a growing concern over the safety of the light sources such as LEDs with peak emissions in the blue light range $(400-490 \mathrm{~nm})$. Recent studies aimed at investigating the effect of exposure to light emitted from electronic device on human skin cells, shows that even short exposures can increase the generation of reactive oxygen species. However, the biological effects of either long-term or repeated exposures are not fully known, yet. Furthermore, there are reports indicating that frequent exposure to visible light spectrum of the selfie flashes may cause skin damage and accelerated skin ageing. In this paper we have addressed the different aspects of potential effects of exposure to the light emitted from smartphones' digital screens as well as smartphones' photoflashes on premature aging of the human skin. Specifically, the effects of blue light on eyes and skin are discussed. Based on current knowledge, it can be suggested that changing the spectral output of LED-based smartphones' flashes can be introduced as an effective method to reduce the adverse health effects associated with exposure to blue light.

Keywords

Smartphones, Mobile Phones, Selfies, Skin Damage, Skin Aging, Blue Light
\end{abstract}

\section{Introduction}

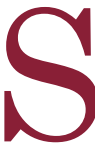
ince the creation of life on the Earth, our skin has been exposed to different levels of visible and invisible light. Over the past decades, due to inevitable consequences of industrialization, modern life and major changes in the life style of large segments of the population of developing and developed countries, humans are not exposed to adequate levels of natural light during the day but are overexposed to relatively high levels of artificial light at night. Recent advances in the technology of light-emitting diodes (LEDs) have led to
${ }^{1}$ Ionizing and Non-ionizing Radiation Protection

Research Center (INIR-

PRC), Shiraz University

of Medical Sciences,

Shiraz, Iran

2Student Research

Committee, School of

Rehabilitation, Shiraz

University of Medical Sci-

ences, Shiraz, Iran

${ }^{3}$ Student Research

Committee, School of

Medicine, Shiraz Univer-

sity of Medical Sciences,

Shiraz, Iran

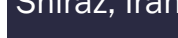

*Corresponding author: S. A. R. Mortazavi Medical Student

School of Medicine, Imam Hossein Square, Shiraz, Iran

E-mail: a.mortazavi.72@ gmail.com

Received: 28 June 2016 Accepted: 20 July 2016 
their widespread use in lighting. Low energy consumption, small size and long lifetime are among the basic advantages of LEDs over the old high energy consuming incandescent light sources. LEDs are currently used in a wide variety of applications ranged from traffic signals to camera and smartphone flashes. The widespread application of LEDs and the rapidly increasing use of smartphones, tablets, laptops and desktop computers have led to a growing concern over the safety of these light sources which their peak emission lies in the blue region (400-490 $\mathrm{nm}$ ).

Reviewing the studies aimed at investigating the effect of exposure to light emitted from electronic devices on human skin cells, it can shed some light on the dark corners of this challenging issue. Austin et al. have recently exposed the AG13145 fibroblasts to electronic devices generated light (EDGL) for 1 hour at a distance of $1 \mathrm{~cm}$ and measured changes in reactive oxygen species (ROS) generation, apoptosis, and necrosis. Their experiment showed that even short exposures can increase the generation of reactive oxygen species. However, the biological effects of either longterm or repeated exposures have not been fully known, yet [1]. Moreover, the link between aging and oxidative stress is well documented [2]. Skin is a major target of oxidative stress [3] and it is widely accepted that extrinsic skin aging can be caused by oxidative stress. Reactive oxygen species (ROS) can be among the cardinal causes of aging is due to these points that not only the production of ROS increase with age, but also the ability of human skin cells to repair DNA damage steadily decreases with aging [4]. Oxidative stress on skin plays a major role in the aging process. This is true for intrinsic aging and even more for extrinsic aging.

Over the past several years, our laboratories at the Ionizing and Non-ionizing Radiation Protection Research Center (INIRPRC) have expanded their focus on studying the health effects of exposure to some common and/or occupational sources of non-ionizing electromagnetic fields (EMFs) such as cellular phones [5-15], mobile base stations [16], mobile phone jammers [17-19], laptop computers [20], radars [6], dentistry cavitrons [21], MRI [22-24], Wi-Fi routers [25] and different coils $[26,27]$. Furthermore, the adverse biological effects of exposure to short-wavelength visible light in blue region either in humans or other living organisms were among our research priorities [28].

\section{The Blue Region of Visible Light \\ I. Blue Light Effects on Eye}

Exposure to blue light is a factor to be linked to photoreceptor damage [29]. Seiler et al. have shown that continuous exposure of albino rats to moderate levels of blue light for 2-5 days removes most of the photoreceptors [30]. In another study, rats were exposed to blue light $\left(400-480 \mathrm{~nm}, 0.7 \mathrm{~W} / \mathrm{m}^{2}\right)$ for $6 \mathrm{~h}$. This study demonstrated that exposure to diffuse blue light caused an uneven distribution of damage in the retina [31]. Rukmini et al. have also reported that in glaucomatous eyes, exposure to high-irradiance blue light reduced the pupillary light reflex and was associated with greater visual field loss and optic disc cupping [32].

\section{Blue Light Effects on Skin}

Sarah Knapton, the Science Editor of the Telegraph in a report entitled "Selfies can age the skin and cause wrinkles, warn dermatologists" that is published on June 17, 2016 reported that dermatologists now believe that due to detrimental effects of irradiation with electromagnetic fields (EMFs) and visible light (VL), frequent exposure to VL and EMFs produced by smartphones can lead to skin damage and accelerated ageing (promoting wrinkles).

Although a large body of evidence indicates that visible light is not safe for the skin, a widely-believed misconception is that exposure to this part of the light cannot lead to any detrimental effects in human skin. It should be 
noted that the complex issue of effective protection of human skin from sun exposure is a challenging issue due to unknown aspects of the interaction between visible light and human tissues [33]. As phototherapy with visible light has become popular in dermatological practice, some researchers have reported that blue light does not cause DNA damage or premature photo-aging and claimed that shortterm application of visible blue light in dermatological practice is safe [34]. Furthermore, it has been reported that exposure of human skin to moderate levels of blue light induced a significant increase in the formation of enzyme-independent cutaneous NO and NO-dependent local biological responses (increased blood flow). These researchers concluded that in contrast to UVA, blue-light-induced NO generation can be used in the treatment of systemic and local hemodynamic disorders which are linked to impaired physiological NO production or bioavailability [35].

Although phototherapy with blue light has become important in the treatment of many dermatologic conditions [36] and even some reports indicate that exposure to blue light can be used in the treatment of superficial skin carcinomas in humans [37], substantial evidence now shows that in a similar pattern to that caused by exposure to infrared (IR) or ultraviolet (UV), at high level, exposure to blue-violet light can be associated with some adverse effects in human skin [38] .

\section{Xenon Photoflashes Versus LEDs}

The xenon flash tubes which are widely used in all types of photography and white light-emitting diodes (LED) are among the main available digital camera photoflashes. While xenon flash is globally used in both film cameras and stand-alone digital still cameras (DSCs) due to its higher level of brightness, white LED flashes are preferred in most camera phones [39]. Although, there are numerous advantages for the use of LEDs over Xenon flashes, the peak spectral intensity of LEDs lies in the blue region [40].

\section{Does Using a Monopod Help?}

If we assume that the power output of the smartphones' photoflashes is constant at any distance (the distance between the subject and camera), when someone uses a monopod, the intensity of light reaching the skin can be significantly reduced. It is worth noting that according to the so called "inverse square law" the intensity of light which reaches to a surface from a point source with constant intrinsic luminosity falls off as the square of the distance from the object. This is known as the inverse square law for light intensity.

\section{Conclusion}

Human skin has been exposed to different levels of light since the creation of life. Interestingly, over the past decades, due to consequences of modern life, humans are not exposed to adequate levels of natural light during the day but are overexposed to relatively high levels of artificial light at night. Exposure to light emitted from electronic devices on human skin cells, even in case of short exposures, can increase the generation of reactive oxygen species. Skin is a major target of oxidative stress and the link between aging and oxidative stress is well documented. Especially, extrinsic skin aging can be caused by oxidative stress. Moreover, LEDs are among the main available digital camera photoflashes. Although, there are numerous advantages for the use of LEDs over Xenon flashes, the peak spectral intensity of LEDs lies in the blue region. Current data show that exposure to blue light can lead to different levels of damage in human eyes and skin. Changing the spectral output of LED-based smartphones' flashes can be introduced as an effective method for reducing the adverse health effects associated with exposure to blue light.

\section{Acknowledgment}

This study was supported by the Ionizing 
and Non-ionizing Radiation Protection Research Center (INIRPRC), Shiraz University of Medical Sciences (SUMS), Shiraz, Iran.

\section{Conflict of Interest}

None Declared

\section{References}

1. Austin E, Huang A, Adar T, Wang E, Jagdeo J. Electronic device generated light increases reactive oxygen species in human fibroblasts. Lasers Surg Med. 2018 doi: 10.1002/lsm.22794.

2. Rinnerthaler M, Bischof J, Streubel MK, Trost A, Richter K. Oxidative stress in aging human skin. Biomolecules. 2015;5:545-89. doi: 10.3390/ biom5020545. PubMed PMID: 25906193; PubMed Central PMCID: PMC4496685.

3. Kohen R. Skin antioxidants: their role in aging and in oxidative stress--new approaches for their evaluation. Biomed Pharmacother. 1999;53:181-92. doi: 10.1016/S0753-3322(99)80087-0. PubMed PMID: 10392290.

4. Poljsak B, Dahmane RG, Godic A. Intrinsic skin aging: the role of oxidative stress. Acta Dermatovenerol Alp Pannonica Adriat. 2012;21:33-6. PubMed PMID: 23000938.

5. Mortazavi SM, Motamedifar M, Namdari G, Taheri M, Mortazavi AR, Shokrpour N. Non-linear adaptive phenomena which decrease the risk of infection after pre-exposure to radiofrequency radiation. Dose Response. 2014;12:233-45. doi: 10.2203/ dose-response.12-055.Mortazavi. PubMed PMID: 24910582; PubMed Central PMCID: PMC4036396.

6. Mortazavi SM, Taeb S, Dehghan N. Alterations of visual reaction time and short term memory in military radar personnel. Iran J Public Health. 2013;42:428-35. PubMed PMID: 23785684; PubMed Central PMCID: PMC3684731.

7. Mortazavi SM, Rouintan MS, Taeb S, Dehghan N, Ghaffarpanah AA, Sadeghi Z, et al. Human shortterm exposure to electromagnetic fields emitted by mobile phones decreases computer-assisted visual reaction time. Acta Neurol Belg. 2012;112:171-5. doi: 10.1007/s13760-012-0044-y. PubMed PMID: 22426673.

8. Mortazavi S, Mosleh-Shirazi M, Tavassoli A, Taheri M, Mehdizadeh A, Namazi S, et al. Increased Radioresistance to Lethal Doses of Gamma Rays in Mice and Rats after Exposure to Microwave Radiation Emitted by a GSM Mobile Phone Simulator. Dose Response. 2013;11:281-92. doi: 10.2203/ dose-response.12-010.Mortazavi. PubMed PMID:
23930107; PubMed Central PMCID: PMC3682203.

9. Mortazavi S, Mosleh-Shirazi M, Tavassoli A, Taheri M, Bagheri Z, Ghalandari R, et al. A comparative study on the increased radioresistance to lethal doses of gamma rays after exposure to microwave radiation and oral intake of flaxseed oil. International Journal of Radiation Research. 2011;9:9.

10. Mortavazi S, Habib A, Ganj-Karami A, SamimiDoost R, Pour-Abedi A, Babaie A. Alterations in TSH and Thyroid Hormones following Mobile Phone Use. Oman Med J. 2009;24:274-8. doi: 10.5001/omj.2009.56. PubMed PMID: 22216380; PubMed Central PMCID: PMC3243874.

11. Mortazavi SM, Daiee E, Yazdi A, Khiabani K, Kavousi $A$, Vazirinejad $R$, et al. Mercury release from dental amalgam restorations after magnetic resonance imaging and following mobile phone use. Pak J Biol Sci. 2008;11:1142-6. PubMed PMID: 18819554.

12. Mortazavi SM, Ahmadi J, Shariati M. Prevalence of subjective poor health symptoms associated with exposure to electromagnetic fields among university students. Bioelectromagnetics. 2007;28:32630. doi: 10.1002/bem.20305. PubMed PMID: 17330851.

13. Mortazavi S, Motamedifar M, Namdari G, Taheri M, Mortazavi A. Counterbalancing immunosuppression-induced infections during long-term stay of humans in space. Journal of Medical Hypotheses and Ideas. 2013;7:8-10.

14. Mortazavi SM, Atefi M, Kholghi F. The pattern of mobile phone use and prevalence of self-reported symptoms in elementary and junior high school students in shiraz, iran. Iran J Med Sci. 2011;36:96103. PubMed PMID: 23358105; PubMed Central PMCID: PMC3556746.

15. Mortazavi SM, Mahbudi A, Atefi M, Bagheri S, Bahaedini $N$, Besharati $A$. An old issue and a new look: electromagnetic hypersensitivity caused by radiations emitted by GSM mobile phones. Technol Health Care. 2011;19:435-43. doi: 10.3233/THC2011-0641. PubMed PMID: 22129944.

16. Mortazavi S. Safety issues of mobile phone base stations. Journal of Biomedical Physics and Engineering. 2013;3.

17. Mortazavi S, Parsanezhad M, Kazempour M, Ghahramani $P$, Mortazavi $A$, Davari $M$. Male reproductive health under threat: Short term exposure to radiofrequency radiations emitted by common mobile jammers. J Hum Reprod Sci. 2013;6:124-8. doi: 10.4103/0974-1208.117178. PubMed PMID: 24082653; PubMed Central PMCID: PMC3778601.

18. Rafati A, Rahimi S, Talebi A, Soleimani A, Haghani 
M, Mortazavi SM. Exposure to Radiofrequency Radiation Emitted from Common Mobile Phone Jammers Alters the Pattern of Muscle Contractions: an Animal Model Study. J Biomed Phys Eng. 2015;5:133-42. PubMed PMID: 26396969; PubMed Central PMCID: PMC4576874.

19. Shekoohi Shooli F, Mortazavi SA, Jarideh S, Nematollahii S, Yousefi F, Haghani M, et al. Short-Term Exposure to Electromagnetic Fields Generated by Mobile Phone Jammers Decreases the Fasting Blood Sugar in Adult Male Rats. J Biomed Phys Eng. 2016;6(1):27-32. PubMed PMID: 27026952; PubMed Central PMCID: PMCPMC4795326.

20. Mortazavi SMJ, Tavassoli A, Ranjbari F, Moammaiee P. Effects of laptop computers' electromagnetic field on sperm quality. Journal of Reproduction \& Infertility. 2010;11(4):251-8.

21. Mortazavi SM, Vazife-Doost S, Yaghooti M, Mehdizadeh S, Rajaie-Far A. Occupational exposure of dentists to electromagnetic fields produced by magnetostrictive cavitrons alters the serum cortisol level. J Nat Sci Biol Med. 2012;3:60-4. doi: 10.4103/0976-9668.95958. PubMed PMID: 22690053; PubMed Central PMCID: PMC3361780.

22. Mortazavi SM, Neghab M, Anoosheh SM, Bahaeddini N, Mortazavi G, Neghab P, et al. High-field MRI and mercury release from dental amalgam fillings. Int J Occup Environ Med. 2014;5:101-5. PubMed PMID: 24748001.

23. Mortazavi SM, Daiee E, Yazdi A, Khiabani K, Kavousi $A$, Vazirinejad $R$, et al. Mercury release from dental amalgam restorations after magnetic resonance imaging and following mobile phone use. Pak J Biol Sci. 2008;11:1142-6. PubMed PMID: 18819554.

24. Mortazavi G, Mortazavi SM. Increased mercury release from dental amalgam restorations after exposure to electromagnetic fields as a potential hazard for hypersensitive people and pregnant women. Rev Environ Health. 2015;30:287-92. doi: 10.1515/ reveh-2015-0017. PubMed PMID: 26544100.

25. Mahmoudi R, Mortazavi S, Safari S, Nikseresht M, Mozdarani $\mathrm{H}$, Jafari M, et al. Effects of microwave electromagnetic radiations emitted from common Wi-Fi routers on rats' sperm count and motility. International Journal of Radiation Research. 2015;13:363-8.

26. Haghnegahdar $A$, Khosrovpanah $H$, AndishehTadbir A, Mortazavi G, Saeedi Moghadam M, Mortazavi S, et al. Design and fabrication of helmholtz coils to study the effects of pulsed electromagnetic fields on the healing process in periodontitis: preliminary animal results. J Biomed Phys Eng.
2014;4:83-90. PubMed PMID: 25505775; PubMed Central PMCID: PMC4258865.

27. Paknahad M, Shahidi S, Mortazavi SMJ, Mortazavi G, SAEEDI MM, DEHGHANI NA. The effect of pulsed electromagnetic fields on microleakage of amalgam restorations: an in vitro study. Shiraz EMed J. 2016;17(e32329).

28. Mortazavi SM, Mortazavi SA, Habibzadeh P, Mortazavi G. Is it Blue Light or Increased Electromagnetic Fields which Affects the Circadian Rhythm in People who Use Smartphones at Night. Iran J Public Health. 2016;45:405-6.

29. Tosini G, Ferguson I, Tsubota K. Effects of blue light on the circadian system and eye physiology. Mol Vis. 2016;22:61-72. PubMed PMID: 26900325; PubMed Central PMCID: PMC4734149.

30. Seiler MJ, Liu OL, Cooper NG, Callahan TL, Petry HM, Aramant RB. Selective photoreceptor damage in albino rats using continuous blue light. $A$ protocol useful for retinal degeneration and transplantation research. Graefes Arch Clin Exp Graefes Arch Clin Exp Ophthalmol. 2000;238(7):599-607. PubMed PMID: 10955662.

31. Wu J, Chen E, Soderberg PG. Failure of ascorbate to protect against broadband blue light-induced retinal damage in rat. Graefes Arch Clin Exp Ophthalmol. 1999;237:855-60. PubMed PMID: 10502061.

32. Rukmini AV, Milea D, Baskaran M, How AC, Perera SA, Aung T, et al. Pupillary Responses to HighIrradiance Blue Light Correlate with Glaucoma Severity. Ophthalmology. 2015;122:1777-85. doi: 10.1016/j.ophtha.2015.06.002.

33. Chiarelli-Neto 0, Ferreira AS, Martins WK, Pavani $C$, Severino D, Faiao-Flores $F$, et al. Melanin photosensitization and the effect of visible light on epithelial cells. PLOS One. 2014;9:e113266. doi: 10.1371/journal.pone.0113266. PubMed PMID: 25405352; PubMed Central PMCID: PMC4236153.

34. Kleinpenning MM, Smits T, Frunt MH, van Erp PE, van de Kerkhof PC, Gerritsen RM. Clinical and histological effects of blue light on normal skin. Photodermatol Photoimmunol Photomed. 2010;26:1621. doi: 10.1111/j.1600-0781.2009.00474.x. PubMed PMID: 20070834.

35. Oplander C, Deck A, Volkmar CM, Kirsch M, Liebmann J, Born M, et al. Mechanism and biological relevance of blue-light (420-453 $\mathrm{nm}$ )-induced nonenzymatic nitric oxide generation from photolabile nitric oxide derivates in human skin in vitro and in vivo. Free Radic Biol Med. 2013;65:1363-77. doi: 10.1016/j.freeradbiomed.2013.09.022.

36. Walker DP, Vollmer-Snarr HR, Eberting CL. Ocu- 
lar hazards of blue-light therapy in dermatology. $J$ Am Acad Dermatol. 2012;66:130-5. doi: 10.1016/j. jaad.2010.11.040. PubMed PMID: 21536341.

37. Sparsa A, Faucher K, Sol V, Durox H, Boulinguez S, Doffoel-Hantz V, et al. Blue light is phototoxic for B16F10 murine melanoma and bovine endothelial cell lines by direct cytocidal effect. Anticancer Res. 2010;30:143-7. PubMed PMID: 20150629.

38. Vandersee S, Beyer M, Lademann J, Darvin ME. Blue-violet light irradiation dose dependently decreases carotenoids in human skin, which indicates the generation of free radicals. Oxid Med Cell Longev. 2015;2015:579675. doi: 10.1155/2015/579675. PubMed PMID: 25741404; PubMed Central PMCID: PMC4337113.

39. LEDs Magazine. Implementing LED flash in camera phones.April 2005. Available From: http://www ledsmagazine com/features/2/4/1/1,(4 pages)

40. Kimme F, Brick P, Chatterjee S, Tran QK. Optimized flash light-emitting diode spectra for mobile phone cameras. Appl Opt. 2013;52:8779-88. doi: 10.1364/ A0.52.008779. PubMed PMID: 24513943. 